\title{
Early Pulmonary Manifestations of Dermatomyositis: A Case Report
}

\author{
Mahnaz Mozdourian ${ }^{1}$ and Zahra Javidarabshahi ${ }^{1, *}$ \\ ${ }^{1}$ Pulmonologist, Lung Diseases Research Center, Mashhad University of Medical Sciences, Mashhad, Iran \\ * Corresponding author: Zahra Javidarabshahi, Lung Diseases Research Center, Mashhad University of Medical Sciences, Mashhad, Iran. \\ Email: mozdorianmh@mums.ac.ir
}

Received 2020 October 24; Revised 2020 November 04; Accepted 2020 November 14.

\begin{abstract}
Introduction: Dermatomyositis (DM) is an idiopathic connective tissue disease with a wide range of systemic manifestations. Progressive proximal skeletal muscle weakness and symmetric and inflammatory infiltrates are the main clinical and histological features of DM.

Case Presentation: In this study, we report the case of a 43-year-old male with DM and early pulmonary manifestations. A $50 \mathrm{mg} / \mathrm{g}$ daily dose of prednisolone was administered for the case. The symptoms of muscle weakness improved after 2 weeks. Commonly, pulmonary diseases occur after the occurrence of other connective tissue diseases. However, in the present case, pulmonary manifestations were observed many years before the incidence of DM symptoms.

Conclusion: The DM with pulmonary involvement is associated with worse outcomes; accordingly, a higher rate of mortality is reported among these patients. Based on the literature, the neutrophil-lymphocyte ratio and platelet-lymphocyte ratio are reliable markers for the diagnosis of DM, along with lung problems.
\end{abstract}

Keywords: Dermatomyositis, Interstitial lung disease, Prednisolone, Pulmonary involvement

\section{Introduction}

Dermatomyositis (DM) is an idiopathic connective tissue disease with a long-term duration, which may appear with or without inflammatory myositis (1). The incidence of DM is 1 per 100,000 mostly appearing after the fifth decade of life (2). It seems that DM is an autoimmune reaction against skin and muscles (3). The disease includes a wide range of systemic manifestations, such as vasculopathy, muscular inflammation, interstitial lung disease (ILD), and muscular and skin involvement (e.g., cutaneous calcifications) (1). The pain and weakness of the proximal muscles are the muscle symptoms of DM. Erythema (over the face, neck, and upper trunk), heliotropic rash, Gottron papules, and rash are the most common skin manifestations. Photosensitivity and pruritus are other reported cutaneous symptoms (3).

Some common symptoms of DM include nail changes, such as capillary telangiectasia along the distal nail fold, periungual erythema, and hyperkeratosis. Moreover, calcinosis or hyperkeratotic lesions on the fingers (Mechanic's hand) are the rare cutaneous symptoms of DM. Calcinosis has been reported in up to $20 \%$ of adults with DM (4). The disease is also associated with malignancies, including breast, ovary, lung, and colon cancers.

The presence of a purple-red discoloration of the upper eyelids with or without eyelid edema is another possible symptom of DM. Commonly, dysphagia appears over time and should be considered a sign of disease progression or underlying malignancy. The DM has various symptoms with no specific sequence, and the presence of all symptoms is not observed in all cases. The clinical manifestations of DM and their severity are different from patient to patient. Moreover, an immune response to viral infections, drugs, or malignancies may lead to DM. The most common therapeutic challenges in DM patients are lung problems (1). The DM with pulmonary involvement is associated with worse outcomes (5). In this study, we report a case of DM with pulmonary manifestations appearing many years before the incidence of DM symptoms.

\section{Case Presentation}

A 43-year-old male complained of weakness of the lower limbs (i.e., polymyositis), progressive shortness of breath, dry cough, and orthopnea. Over the past year, he lost weight due to dysphagia. The medical history of the patient showed diabetes over the past 2 years, cough, and shortness of breath. The results of a computed tomography (CT) scan showed lung involvement, and the patient himself did not refer for follow-up visits. He had a history of using neutral protamine Hagedorn and regular insulin, and he has recently reported muscle weakness.

The case was reported with a body temperature of $37.2^{\circ} \mathrm{C}$, oxygen saturation of $86 \%$, pulse rate of 100 beats per minute, respiratory rate of 30 breaths per minute, and blood pressure of 105/80 $\mathrm{mmHg}$. In the eye examination, there was a pale appearance in the palpebral conjunctiva. The prominent jugular 


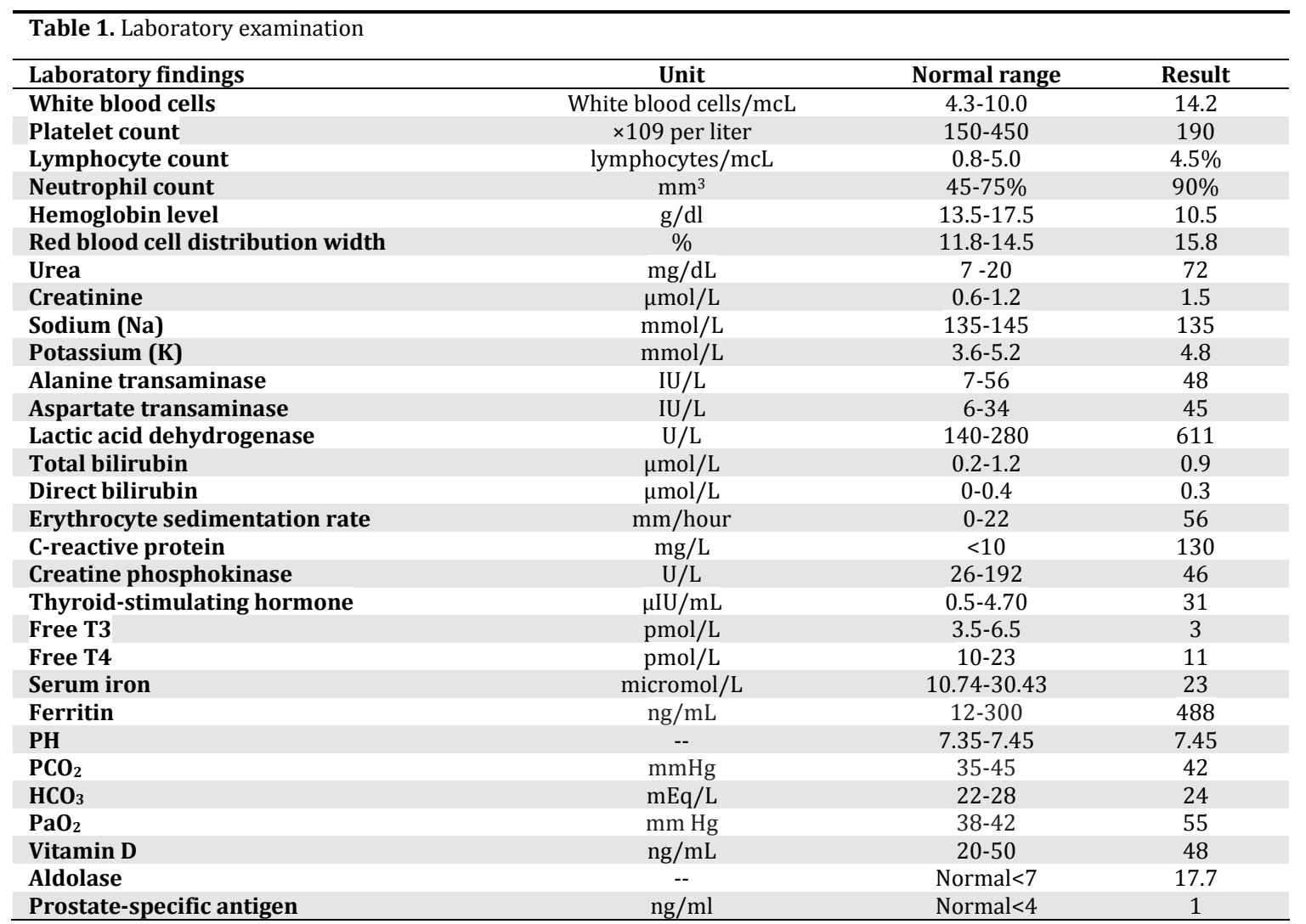

veins were also confirmed. Tachycardia with prominent pulmonary sound was confirmed by listening to the heart sounds. In lung auscultation, fine crackles were observed in the lower third of the lungs. The abdominal examination and deep tendon reflexes were normal. In the examination of the lower limbs, there was a reduction in the muscle force (score: 3.5 ).

The results of laboratory examinations are shown in Table 1. Hepatitis B surface antigen, hepatitis C virus antibody, and human immunodeficiency virus antibody were negative. The patient had lung symptoms and was referred for a lung CT scan. A high-resolution CT of the lung showed bilateral ground-glass opacities. Due to lung involvement, bronchoscopy was conducted showing that the patient did not have an endobronchial lesion.

Bronchial lavage was performed with a negative report of malignancy. Moreover, the culture tests for mycobacterium tuberculosis, fungi, and bacteria were negative. Transbronchial lung biopsy showed a mixed inflammation of alveolus. Pulmonary manifestations were observed many years before the incidence of DM symptoms. The echocardiogram showed normal left ventricular function and mild tricuspid regurgitation. The tricuspid regurgitation peak gradient was $35 \mathrm{mmHg}$, and systolic pulmonary artery pressure was $50 \mathrm{mmHg}$. No pericardial effusion was reported.

Rheumatology tests were requested due to lung involvement and myopathy. Antinuclear antibody (ANA), anti-double stranded DNA, and ANA profile were normal. Anti-signal recognition particle, antibodies against $\mathrm{Ku}$ antigen, anti-Mi2, and antialanyl-tRNA synthetase were positive. In addition, Anti-PM/Scl antibodies and anti-Jo1 were borderline.

Due to the symptoms of muscle weakness, positive rheumatological tests, and high diagnostic suspicion of DM accompanied by malignancies, complete abdominal ultrasound, endoscopy, and colonoscopy were performed for the diagnosis of underlying malignancy. The obtained results were normal ruling out the probability of malignancy. Electromyography was performed due to muscle weakness, and nerve conduction velocity showed myopathic disorder.

According to the above-mentioned conditions, the patient was diagnosed with DM and lung involvement. Therefore, a $50 \mathrm{mg} / \mathrm{g}$ daily dose of prednisolone was administered. The muscle symptoms improved after 2 weeks, and the CT scan showed partial improvement in pulmonary infiltration after a month (figures 1 , 2 , and 3).

\section{Discussion}

The mechanism of DM has not yet been completely perceived. The DM has an autoimmune nature due to inflammatory tissue reaction, vasculitis, and presence of $\mathrm{T}$ cell-mediated 
myotoxicity (2). Most of the time, the disease develops during or after the appearance of muscle inflammation. It is associated with higher levels of fever and joint involvement (6). Progressive proximal skeletal muscle weakness and symmetric and inflammatory infiltrates are the main clinical and histological features of DM (2). The systemic manifestations of DM are often complicated, including a wide range. In the majority of reported cases, dyspnea and respiratory problems are the most common problems of DM $(2,7)$.

Firstly, endothelial antigens are affected by DM. The activation of the complement system and deposition of immune complexes in the capillary bed occur before inflammatory or structural muscle changes. There are several autoantibodies against nuclear and cytoplasmic antigens in individuals with inflammatory myopathies the role of which is still uncertain. Moreover, these autoantibodies are observed in less than $25 \%$ of patients with DM (8).
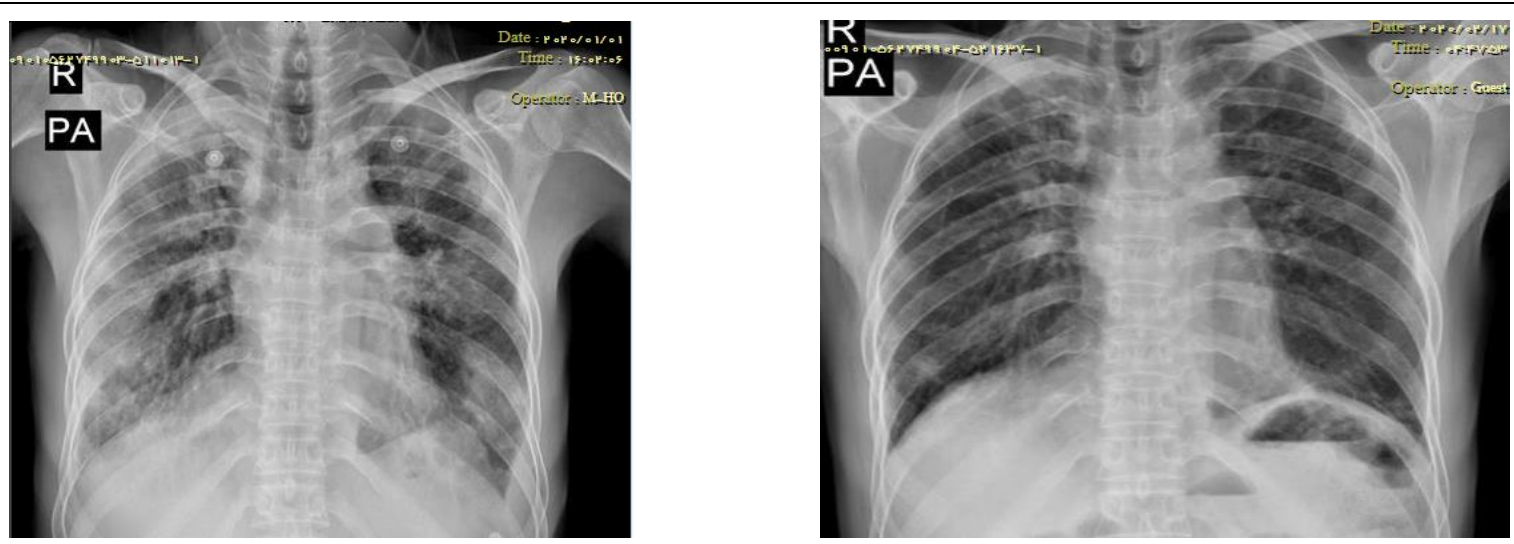

Figure 1. Chest X-ray before and 2 weeks after treatment
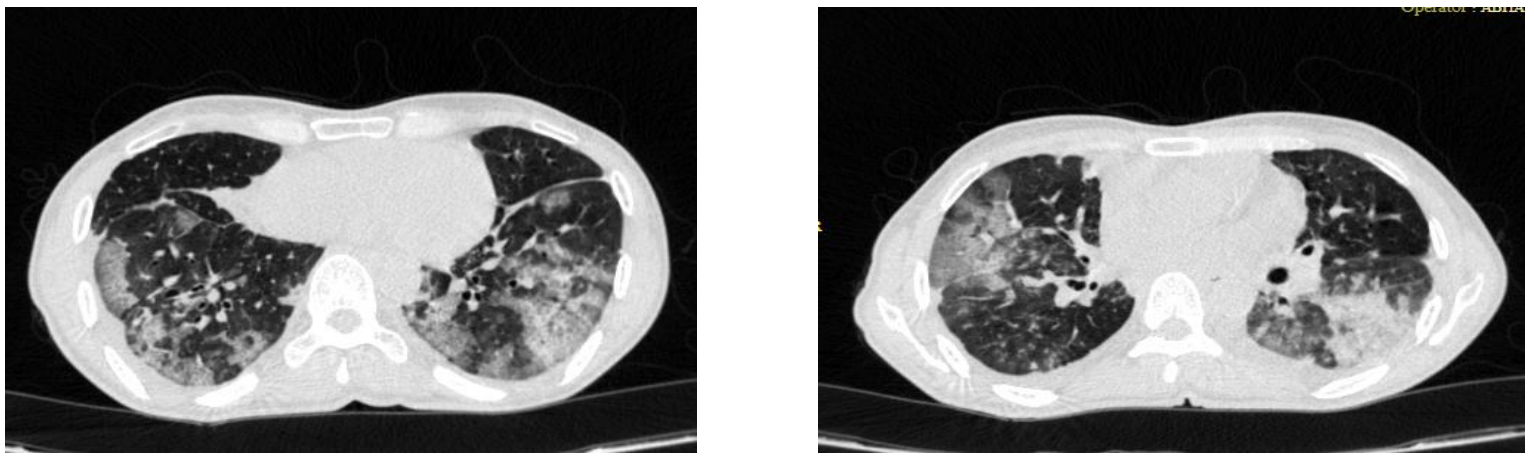

Figure 2. Computed tomography scan of patient in ground-glass and consolidation view in initiation of treatment
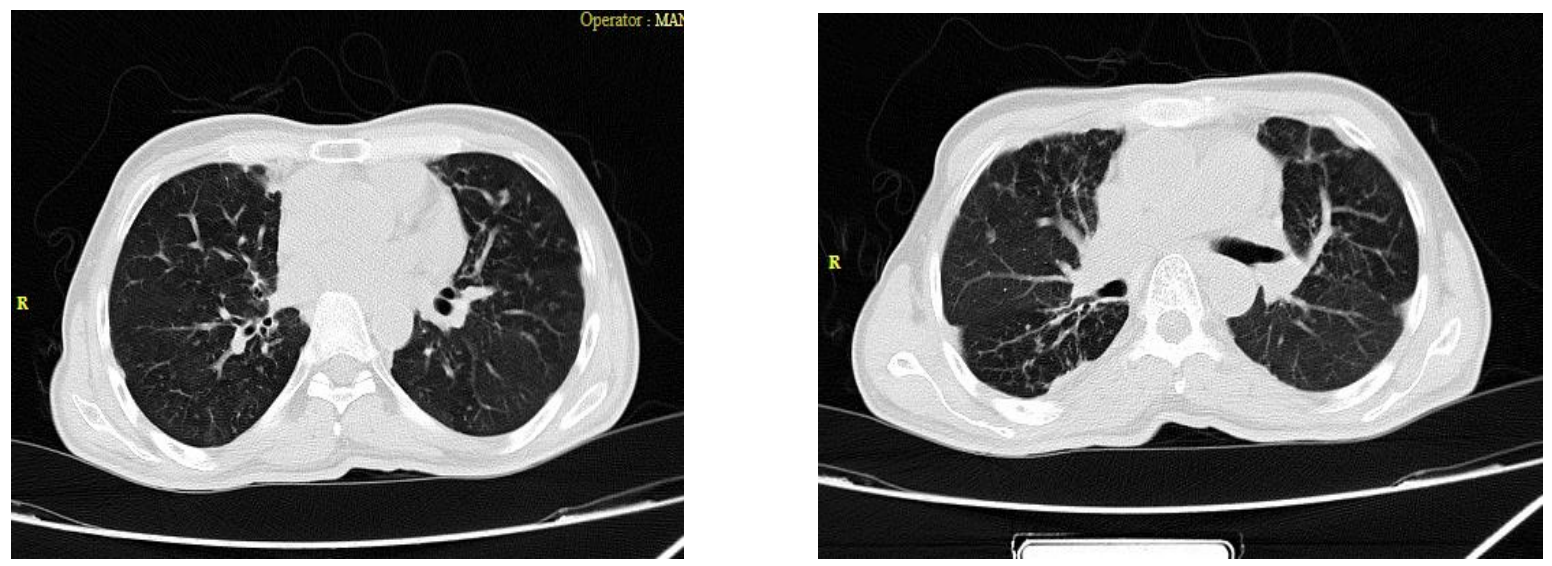

Figure 3. Computed tomography scan of patient in ground-glass and consolidation view after one month of treatment 
The risk of malignancy is higher among patients with DM in comparison to that reported for other inflammatory myopathies; accordingly, the risk of neoplasm, especially ovary, lung, pancreas, stomach, and colorectal cancers, is three times higher in DM patients in comparison to that of other diseases (9). The most common therapeutic challenge in DM patients is ILD (1). Aspiratory or ventilatory insufficiency is another lung problem, which may be observed in the next step of the disease due to the complication of striated muscle dysfunction. Lung diseases may also occur due to the treatment of DM by drug-induced interstitial pneumonitis.

Wendel et al. reported two DM patients with pulmonary involvement suffering from severe debilitating calcinosis. One patient showed a rapid deterioration of pulmonary function, which was resistant to immunosuppressants (10). The DM with pulmonary involvement is associated with worse outcomes; accordingly, a higher rate of mortality has been reported among these patients (5).

The ILD is observed in subjects with DM; however, it is not an original criterion for the diagnosis of the disease (6). Pulmonary complications are the main important determinants of the outcomes of DM. A restrictive ventilatory defect may be a consequence of pulmonary diseases or be caused by extrapulmonary conditions, such as respiratory muscle weakness, calcinosis in the chest wall, or pleural disease (11).

Commonly, pulmonary diseases occur after the occurrence of other connective tissue diseases (12). However, in the present case, pulmonary manifestations were observed many years before the incidence of DM symptoms. Nevertheless, in other similar reported cases, pulmonary diseases were diagnosed after the occurrence of DM $(3,13)$.

There is an association between DM and risk of rapidly progressive fatal ILD (3). In a study carried out by Fujita et al., DM and lung cancer were concomitantly diagnosed (14). Although the tumor may be diagnosed before or simultaneously, the majority of malignancies are diagnosed after DM. A coexistence between DM and lung cancer is reported in a few studies (15). Based on the evidence, the presence of DM is introduced as a paraneoplastic manifestation of lung cancer (3).

Based on the literature, the neutrophillymphocyte ratio (NLR) is considered a reliable marker of inflammation, which is correlated with other inflammatory markers, such as C-reactive protein (CRP) and erythrocyte sedimentation rate (ESR) $(16,17)$. A study carried out by Jing Zou et al. assessed the mortality rate of acute ILD in clinically amyopathic DM patients. The obtained results showed a higher NLR in non-survivors in comparison to that reported for survivors (18).

The results of another study showed higher levels of NLR in DM patients, compared to those reported for healthy controls. The positive correlation between the NLR with CRP and ESR was confirmed in the aforementioned study (19). In the present case, the neutrophil count was $90 \%$. Moreover, the high rates of CRP and ESR were reported for the case. The NLR can be suggested as a potential inflammatory indicator for DM with pulmonary involvement.

The platelet-lymphocyte ratio (PLR) is another indicator in order to distinguish between DM patients with and without ILD (19). The results of previous studies have shown that lower lymphocyte counts are associated with severe disease activity in DM patients $(20,21)$. A higher rate of the PLR in DM patients was reported in a study conducted by Yang et al. (19). Similarly, lymphopenia was observed in the present case; however, the palette count was in the normal range. It is required to carry out future studies to confirm the diagnostic value of the PLR in DM patients with pulmonary involvement. Today, the outcomes of patients with idiopathic inflammatory myopathy have improved due to increasing the number of immunomodulatory treatment approaches; accordingly, the survival rate of the patients increased to higher than 90\% (1).

High-dose corticosteroids are commonly applied for suppressing inflammation and underlying autoimmunity (22). Similarly, steroid therapy is used for patients with DM. In this case report, a $50 \mathrm{mg} / \mathrm{g}$ daily dose of prednisolone was administered. However, corticosteroid therapy is associated with several side effects, such as steroid myopathy, osteoporosis, and obesity. Based on the results of a randomized trial, prednisone therapy in combination with either cyclosporine or methotrexate was more effective with lower side effects than prednisone alone in the treatment of patients with DM (23). In the current study, no side effect was observed after prednisolone therapy.

Commonly, pulmonary diseases occur after the occurrence of other connective tissue diseases. However, in the present case, pulmonary manifestations were observed many years before the incidence of DM symptoms. The DM with pulmonary involvement is associated with worse outcomes. The NLR and PLR are reliable markers for the diagnosis of DM, along with lung problems.

\section{Acknowledgements}

The authors would like to express their gratitude to all individuals who participated in this study.

\section{Footnotes}

Authors' Contribution: Mahnaz Mozdourian conceived the study and contributed to designing the study and drafting the manuscript. Zahra Javid revised and approved the manuscript of the study. Conflict of Interests: The authors declare that there 
is no conflict of interest.

Ethical Approval: Informed consent was obtained from the patient.

Funding/Support: The current study was not financially supported by any institutions.

\section{References}

1. Wendel S, Venhoff N, Frye BC, May AM, Agarwal P, Rizzi M et al. Successful treatment of extensive calcifications and acute pulmonary involvement in dermatomyositis with the JanusKinase inhibitor tofacitinib - A report of two cases. $J$ Autoimmun. 2019;100:131-6. doi: 10.1016/j.jaut.2019.03.003. [PubMed: 30862449].

2. Castro A, Barroso A, Parente B. Dermatomyositis as the first manifestation of a lung tumor. Rev Port Pneumol. 2013; 19(4):179-83. doi: 10.1016/j.rppneu.2012.11.002. [PubMed: 23602006].

3. Papakonstantinou E, Kapp A, Raap U. A mild form of dermatomyositis as a prodromal sign of lung adenocarcinoma: a case report. J Med Case Rep. 2016;10(1):34. doi: 10.1186/s13256-016-0816-8. [PubMed: 26851947].

4. Goldfischer J, Rubin EH. Dermatomyositis with pulmonary lesions. Ann Intern Med. 1959;50(1):194-206. doi: 10.7326/ 0003-4819-50-1-194. [PubMed: 13617837].

5. Chen I-J, Wu YJ, Lin CW, Fan KW, Luo SF, Ho $\mathrm{HH}$ et al. Interstitial lung disease in polymyositis and dermatomyositis. Clin Rheumatol. 2009;28(6):639-46.

6. Hallowell RW, Ascherman DP, Danoff SK, editors. Pulmonary manifestations of polymyositis/dermatomyositis. Semin Respir Crit Care Med. 2014;35(2):239-48. doi: 10.1055/s-00341371528. [PubMed: 24668538].

7. Magro CM, Schaefer JT, Waldman J, Knight D, Seilstad K, Hearne D. Terbinafine-induced dermatomyositis: a case report and literature review of drug-induced dermatomyositis. J Cutan Pathol. 2008;35(1):74-81. doi: 10.1111/j.16000560.2007.00767.x. [PubMed: 18096000].

8. Muscle examination in the evaluation of weakness. UpToDate.Available at: URL: https://www.uptodate.com/ contents/muscle-examination-in-the-evaluation-of weakness; 2012.

9. Hill CL, Zhang Y, Sigurgeirsson B, Pukkala E, Mellemkjaer L, Airio A et al. Frequency of specific cancer types in dermatomyositis and polymyositis: a population-based study. Lancet. 2001;357(9250):96-100. doi: 10.1016/S01406736(00)03540-6. [PubMed: 11197446].

10. Wendel S, Venhoff N, Frye BC, May AM, Agarwal P, Rizzi M, et al. Successful treatment of extensive calcifications and acute pulmonary involvement in dermatomyositis with the Januskinase inhibitor tofacitinib-A report of two cases. J Autoimmun. 2019;100:131-6. doi: 10.1016/j.jaut.2019.03.003. [PubMed: 30862449].

11. Sanner H, Aaløkken TM, Gran JT, Sjaastad I, Johansen B, Flatø B. Pulmonary outcome in juvenile dermatomyositis: a casecontrol study. Ann Rheum Dis. 2011;70(1):86-91. doi: 10.1136/ard.2010.131433. [PubMed: 20805295].

12. Lega JC, Cottin V, Fabien N, Thivolet-Béjui F, Cordier JF.
Interstitial lung disease associated with anti-PM/Scl or antiaminoacyl-tRNA synthetase autoantibodies: a similar condition? J Rheumatol. 2010;37(5):1000-9. doi: 10.3899/ jrheum.090652. [PubMed: 20231208].

13. Beel AJ, Demos DS, Chung A, Liao C, Lui NS. Ground-glass opacity heralding invasive lung adenocarcinoma with prodromal dermatomyositis: a case report. J Cardiothorac Surg. 2018;13(1):20. doi: 10.1186/s13019-018-0705-x. [PubMed: 29415746].

14. Fujita J, Tokuda M, Bandoh S, Yang Y, Fukunaga Y, Hojo S, et al. Primary lung cancer associated with polymyositis/ dermatomyositis, with a review of the literature. Rheumatol Int. 2001;20(2):81-4. doi: 10.1007/s002960000070. [PubMed: 11269538].

15. Collins LG, Haines C, Perkel R, Enck RE. Lung cancer: diagnosis and management. Am Fam physician. 2007;75(1):56-63. [PubMed: 17225705].

16. Ahsen A, Ulu MS, Yuksel S, Demir K, Uysal M, Erdogan M, et al. As a new inflammatory marker for familial Mediterranean fever: neutrophil-to-lymphocyte ratio. Inflammation. 2013; 36(6):1357-62. doi: 10.1007/s10753-013-9675-2. [PubMed: 23794006].

17. Qin B, Ma N, Tang Q, Wei T, Yang M, Fu H, et al. Neutrophil to lymphocyte ratio (NLR) and platelet to lymphocyte ratio (PLR) were useful markers in assessment of inflammatory response and disease activity in SLE patients. Mod Rheumatol. 2016;26(3):372-6. doi: 10.3109/14397595.2015.1091136. [PubMed: 26403379].

18. Zou J, Guo Q, Chi J, Wu H, Bao C. HRCT score and serum ferritin level are factors associated to the 1-year mortality of acute interstitial lung disease in clinically amyopathic dermatomyositis patients. Clin Rheumatol. 2015;34(4):707-14. doi: 10.1007/s10067-015-2866-5. [PubMed: 25609178].

19. Yang W, Wang $\mathrm{X}$, Zhang $\mathrm{W}$, Ying $\mathrm{H}, \mathrm{Xu} \mathrm{Y,} \mathrm{Zhang} \mathrm{J} \mathrm{et} \mathrm{al.}$ Neutrophil-lymphocyte ratio and platelet-lymphocyte ratio are 2 new inflammatory markers associated with pulmonary involvement and disease activity in patients with dermatomyositis. Clin Chim Acta. 2017;465:11-16. doi: 10.1016/j.cca.2016.12.007. [PubMed: 27965019].

20. Uslu AU, Küçük A, Sahin A, Ugan Y, Yılmaz R, Güngör T et al. Two new inflammatory markers associated with Disease Activity Score-28 in patients with rheumatoid arthritis: neutrophil-lymphocyte ratio and platelet-lymphocyte ratio. Int Rheumatic Dis. 2015;18(7):731-5. doi: 10.1111/1756185X.12582. [PubMed: 25900081].

21. Wu Y, Chen Y, Yang X, Chen L, Yang Y. Neutrophil-tolymphocyte ratio (NLR) and platelet-to-lymphocyte ratio (PLR) were associated with disease activity in patients with systemic lupus erythematosus. Int Immunopharmacol. 2016;36:94-9. doi: 10.1016/j.intimp.2016.04.006. [PubMed: 27111516].

22. Marie I. Morbidity and mortality in adult polymyositis and dermatomyositis. Curr Rheumatol Rep. 2012;14(3):275-85. doi: 10.1007/s11926-012-0249-3. [PubMed: 22410829].

23. Ruperto N, Pistorio A, Oliveira S, Zulian F, Cuttica R, Ravelli A et al. Prednisone versus prednisone plus ciclosporin versus prednisone plus methotrexate in new-onset juvenile dermatomyositis: a randomised trial. Lancet. 2016; 387(10019):671-8. doi: 10.1016/S0140-6736(15)01021-1. [PubMed: 26645190]. 